\title{
First Steps in the Construction of the Geometric Machine Model ${ }^{1}$
}

R.H.S. REISER ${ }^{2}$, A.C.R. COSTA ${ }^{3}$, G.P. DIMURO 4 , ESIN, UCPel, Cx.P. 402, 96010140 Pelotas, RS, Brazil.

\begin{abstract}
This work introduces the Geometric Machine (GM) - a computational model for the construction and representation of concurrent and non-deterministic processes, preformed in a synchronized way, with infinite memory whose positions are labelled by the points of a geometric space. The ordered structure of the GM model is based on Girard's Coherence Spaces. Starting with a coherence space of elementary processes, the inductive domain-theoretic structure of this model is step-wise and systematically constructed and the procedure completion ensures the existence of temporally and spatially infinite computations. A particular aim of our work is to apply this coherence-space-based interpretation to the semantic modelling parallelism and distributed computation over array structures.
\end{abstract}

\section{Introduction}

Inspired by the work of Scott [6], this work introduces a constructive and intuitive machine model, with infinite memory, called Geometric Machine (GM). Over the GM ordered structure, we obtain representations of (non-)deterministic processes, labelled by positions of a geometric space, including two special types of parallelism - the temporal and the spacial parallelism. Its most basic notion is that of a coherence relation representing the admissibility of parallelism between two or more basic operations (elementary processes). That relation defines a web over which a coherence space [3] of parallel processes is step-wise and systematically built. In the dual construction, the incoherence relation interprets the condition that models non-determinism. The sequential product and the deterministic sum of parallel and non-deterministic processes are endofunctors in the category $\mathcal{C}$ osp $\mathcal{L}$ in of coherence spaces with linear functions as morphisms.

This paper is divided in four sections. Section 3. introduces the Coherence Space $\mathbb{S}$ of computational states of the GM model, and the Section 4. shows the inductive construction of the machine model itself. The conclusion section presents further work and possible applications of the model.

\footnotetext{
${ }^{1}$ This work receives financial support from CAPES, CNPq and FAPERGS.

${ }^{2}$ PPGC, II, UFRGS, CP 15064, 91501-970 Porto Alegre, RS, e-mail: reiser@atlas.ucpel.tche.br ${ }^{3}$ PPGC, II, UFRGS, CP 15064, 91501-970 Porto Alegre, RS, e-mail: rocha@atlas.ucpel.tche.br ${ }^{4}$ e-mail: liz@atlas.ucpel.tche.br.
} 


\section{The Idea of the Geometric Machine Model}

Firstly, the memory states and possible programs in the GM model are specified. Computational processes are then related to the notion of transformations between computational states. In this paper we consider only uni-dimensional memories, but the model can be easily generalized to consider multi-dimension memories. A computational state is defined as a function $s$ from labels to values, where labels are intuitively understood as points of a geometric space, so that a computational state is an assignment of values to points of the geometric space.

In the following, let $I$ be the set of labels of a geometric space and $V$ be the set of values together with a distinguished element $\sharp$.

Definition 1. A function $s: I \rightarrow V$ in the collection $S=[I \rightarrow V]$ is called a computational state. If $I=\left\{i_{n}\right\}_{n \in \omega}$ then $S=\left\{\left\{s\left(i_{n}\right)=v_{n}\right\}_{\{n \in \omega\}} \mid s \in S\right\}$.

Definition 2. Let $S=[I \rightarrow V]$ be the set of all computational states, $\mathcal{A}=\{p:$ $S \rightarrow V \mid p(s)=v\}$ be the set of computational actions and $\mathcal{A}_{I}=\bigcup\left\{\mathcal{A}_{n}\right\}_{i_{n} \in I}$ be the set of labelled actions. Then, a computational process $\mathbf{p}: S \rightarrow S$ is given by $\mathbf{p}(s)=\left\{p^{(n)}(s) \mid p^{(n)} \in \mathcal{A}_{n} \subseteq \mathcal{A}_{I}\right\}=\left\{v_{n}\right\}_{i_{n} \in I} \in \mathcal{P}^{5}$.

If $I=\left\{i_{n} \mid n \in A=\{0,1,2, \ldots, n\} \subseteq \omega\right\}$, $\mathbf{p}$ is a spatially finite computational process. If $I=\left\{i_{n}\right\}_{n \in \omega}, \mathbf{p}(s)=\left\{p^{(n)}(s)\right\}_{n \in \omega}$ is represented in Figure 1 (a).

Definition 3. For $i \in I$, consider the labelled actions $d^{(m)}, p r^{(m)}: S \rightarrow S \in \mathcal{A}_{m}$ where $\operatorname{pr}^{(m)}(s)=s\left(i_{m}\right)$. An elementary computational process $\mathbf{d}^{(n)}$, visually symbolized in the Figure 1 (b), is a set of labelled actions defined by

$$
\mathbf{d}^{(n)}=\left\{t^{(m)}\right\}_{i_{m} \in I}, \quad \text { such that } t^{(m)}(s)= \begin{cases}p r^{(m)}(s), & \text { if } m \neq n, \\ d^{(n)}(s), & \text { otherwise. }\end{cases}
$$

The function $\left\{p^{(m)}\right\}_{i_{m} \in I}: S \rightarrow S$ defines the identity process, denoted by skip and represented in the Figure 1 (c). $\mathcal{D}_{0}=\left\{\mathbf{d}^{(n)}\right\}_{i_{n} \in I} \subseteq \mathcal{P}$ is the set of all non-identity elementary processes. In addition, $\mathcal{D}=\mathcal{D}_{0} \bigcup\{$ skip $\}$.

Consider $B=\{T, F\}$ as the Boolean set and let $\mathcal{B} \equiv[S \rightarrow B]$ be the set of all computational tests. A function $t \in \mathcal{B}$, pictured in Figure 1 (d), is called a computational test.

Suppose $e, d \in \mathcal{A}, i_{k}, i_{l} \in I$ and $d^{(k)}, e^{(k)}, e^{(l)} \in \mathcal{D}$. Figure 2 presents a graphic representation of the usual algebraic process constructors - deterministic (b) or non-deterministic (c) sums and sequential (a) or parallel (d) products, which are the basic compounding elementary processes operations.

\footnotetext{
${ }^{5} \mathcal{P}$ denoted the set of all computational processes
} 


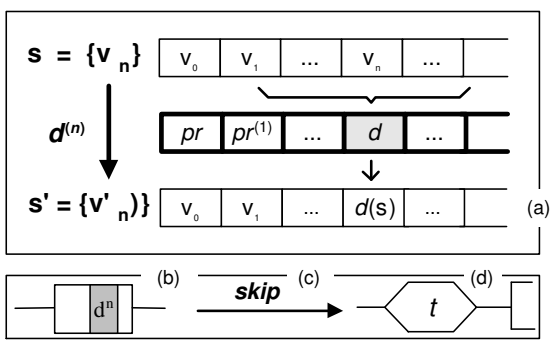

Figure 1: Computational elements.

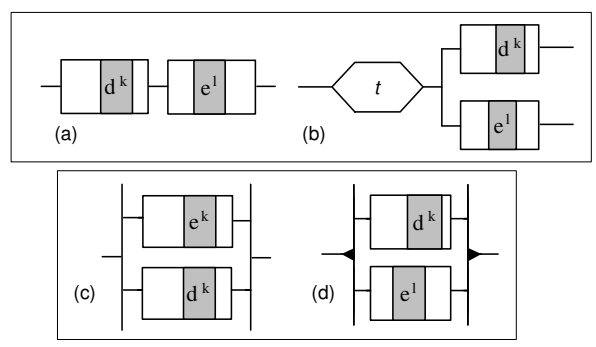

Figure 2: Constructors of processes.

\section{The Coherent Space of Computational States}

A web $\mathbf{W}=\left(W, \approx_{W}\right)$ is a pair consisting of a set $W$ with a symmetric and reflexive relation $\approx_{W}$, called coherence relation. A subset of this web with pairwise consistent elements by the coherence relation $\approx_{W}$ is a coherent subset. The set of coherent subsets of the web $\mathbf{W}$, ordered by the inclusion relation, is called the coherence space $\mathbb{W} \equiv(\operatorname{Coh}(\mathbf{W}), \subseteq)$.

The coherence space $\mathbb{S}$ introduced in this section provides a domain-theoretical formalization for the set $S$ of all computational states of the GM model. For that, consider the flat domain $\mathbb{I}=(\operatorname{Coh}(\mathbf{I}), \subseteq)$ of the labels and let $\mathbf{V}=(\operatorname{Val}, \approx)$ be a web defined by a set Val of value representations, together with a coherence relation $\approx_{\mathbf{V}} \cdot \mathbb{V} \equiv(\operatorname{Coh}(\mathbf{V}), \subseteq)$ denotes the domain of the value representations. For any set of values $V$ we say that $\mathbb{V}$ represents $V$ if $\emptyset \in \operatorname{Coh}(\mathbf{V})$ represents the value $\sharp \in V$ and each nonempty coherence subset models a value $v \neq \sharp, v \in V$.

Consider now $S=\{s: \mathbb{I} \rightarrow \mathbb{V}\}$ as the set of all strict, continuous, stable and linear functions from $\mathbb{I}$ to $\mathbb{V}$. The family of the linear traces $[2,7]$ of such functions determines the coherence space that represents the set of all computational states of the GM model. Let $\left.\mathbf{I} \multimap \mathbf{V}=(|\mathbb{I}| \times|\mathbb{V}|), \approx_{\circ}\right)$ be a web together with the coherence relation $\approx_{-}$given by $(i, v) \approx_{-}\left(i^{\prime}, v^{\prime}\right) \Rightarrow\left(v \cong_{\mathbf{V}} v^{\prime}\right.$ and $\left.\left(v=v^{\prime} \Rightarrow i=i^{\prime}\right)\right)$. The collection of coherence subsets of the web $\mathbf{I} \multimap \mathbf{V}$, ordered under inclusion defines the coherence space $\mathbb{I} \multimap \mathbb{V} \equiv(\operatorname{Coh}(\mathbf{I} \multimap \mathbf{V}), \subseteq)$ of the linear traces of the linear functions from $\mathbb{I}$ to $\mathbb{V}$. In particular, the empty set interprets the linear function which graph is empty.

In the same way, starting with the domain $\mathbb{I} \multimap \mathbb{V}$, we introduce now the definition of the coherent space $\mathbb{S}$ of the computational states. A subset in $\mathbb{I} \multimap \mathbb{V}$ interprets a consistent piece of information. Thus, a computational state can be understood as a collection of linear traces related to linear functions.

The last idea is compatible with the interpretations of non-deterministic computations, i. e. a subset of $\mathbb{I} \multimap \mathbb{V}$ models a possible outcome of a non-deterministic computation. Thus,

Definition 4. Given the domain $\mathbb{I} \multimap \mathbb{V}$ and the trivial coherence relation, the collection $\operatorname{Coh}(\operatorname{Coh}(\mathbf{I} \multimap \mathbf{V}), \approx)$ ordered under inclusion is the coherence space $\mathbb{S}$ of all computational states of the GM model. 
Observe that, the identification $\mathbb{S} \equiv \mathbb{I} \multimap \mathbb{V}$ is hold whenever only deterministic computations are considered. In this case, an element in $\mathbb{I} \multimap \mathbb{V}$ can be modelled as a memory state.

\section{The Coherent Space of Computational Processes}

The inductive construction of the coherence space $\mathbb{D}_{\infty}$ of all processes in $\mathcal{P}$ and the corresponding completion procedure (see Figure 3) provides a domain-theoretical structure for representations of computational processes involving concurrence and non-determinism over the computational states defined in the previous section ${ }^{6}$.

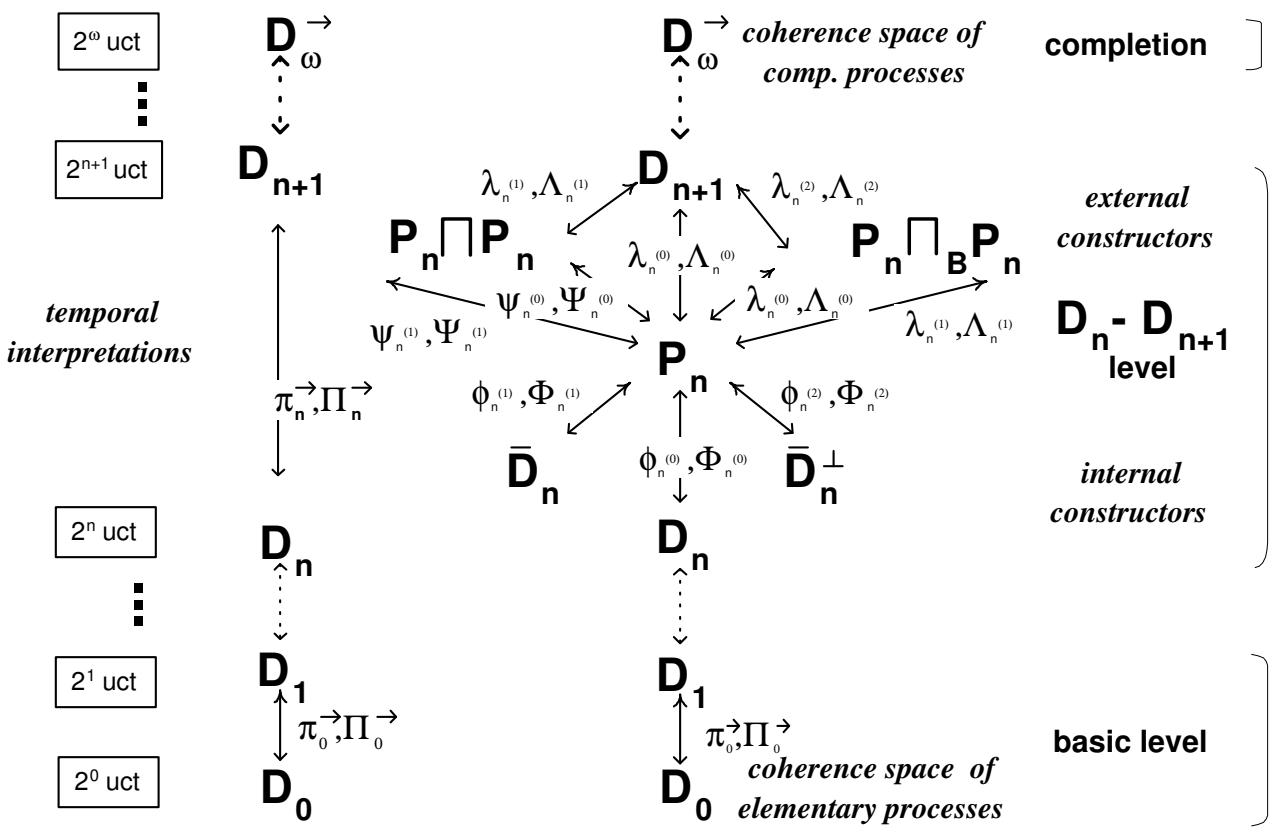

Figure 3: The inductive construction of the coherence space $\mathbb{D}_{\infty}$.

In the following, the definitions of the objects and morphisms related to the basic level $\mathbb{D}_{0}-\mathbb{D}_{1}$ of the inductive construction of $\mathbb{D}_{\infty}$ is introduced. For that, in the rest of paper consider $\left\{d^{(k)}\right\} \equiv \overline{d^{(k)}}$.

Definition 5. Let $\mathbf{D}_{0} \equiv\left(\mathcal{D}_{0} \cong\right)$ be a discrete web defined by the collection of all elementary processes in $\mathcal{D}_{0}$ (Definition 3 ) together with the equality relation. $\mathbb{D}_{0}=\left(\operatorname{Coh}\left(\mathbf{D}_{0}\right), \subseteq\right)$ denotes the coherence space of elementary processes.

In this case, $\operatorname{Coh}\left(\mathbf{D}_{0}\right)=\{\emptyset\} \cup\left\{\overline{d^{(k)}} \mid d^{(k)} \in \mathcal{D}_{0}\right\}$. As each coherent subset $\overline{d^{(k)}} \in \mathbb{D}_{0}$ represents the elementary process $\mathbf{d}^{(k)} \in \mathcal{D}_{0}$, the identity process is

\footnotetext{
${ }^{6}$ The symbol $\infty$ is denoted by $\omega$ in the figures.
} 
represented by the empty set, which constitutes the bottom of the domain $\mathbb{D}_{0}$. The next coherence space in the construction, related with the family $\operatorname{Coh}\left(\mathbf{D}_{0}\right)$, gives interpretation for concurrent computational processes with at most 1 uct ${ }^{7}$.

Definition 6. $\overline{\mathbb{D}}_{0} \equiv\left(\operatorname{Coh}\left(\overline{\mathbf{D}}_{0}\right), \subseteq\right)$ denotes the domain of parallel products of elementary processes. In the web $\overline{\mathbf{D}}_{0} \equiv\left(\overline{\mathcal{D}}_{0}, \approx\right)$, the coherence relation is given by $\overline{d^{(k)}} \approx \overline{e^{(l)}} \Leftrightarrow d^{(k)}=e^{(l)}$ or $k \neq l$. In particular, $\emptyset \approx \overline{d^{(k)}}, \forall \overline{d^{(k)}} \in \overline{\mathbf{D}}_{0}$.

Definition 7. The domain $\overline{\mathbb{D}}_{0}^{\perp} \equiv\left(\operatorname{Coh}\left(\overline{\mathbf{D}}_{0}^{\perp}\right), \subseteq\right)$ of the non-deterministic sum of elementary processes is defined over the complementary web $\overline{\mathbf{D}}_{0}^{\perp} \equiv\left(\overline{\mathcal{D}}_{0}, \approx_{\perp}\right)$ whose coherence relation $\approx_{\perp}$ is the incoherence relation in $\overline{\mathbf{D}}_{0}$.

By the incoherence relation in $\overline{\mathbf{D}}_{0}, \overline{d^{(k)}} \nsim \overline{e^{(l)}} \Leftrightarrow k=l, \overline{d^{(k)}}$ and $\overline{d^{(l)}}$ are said to be incoherent tokens of the web $\overline{\mathbf{D}}_{0}$, which means elementary processes in $\mathcal{D}_{0}$ that conflict in memory access. For instance, the coherent subsets $\left\{\overline{d^{(k)}}, \overline{e^{(k)}}\right\} \in \overline{\mathbb{D}}_{0}^{\perp}$ and $\{\emptyset\},\left\{\emptyset, \overline{f^{(n)}}\right\} \in \overline{\mathbb{D}}_{0}$ interpret the partial processes $\left|d^{(k)}, e^{(k)}\right|$, \|skip $\|$ and $\|$ skip, $f^{(n)} \|$, respectively. See a representation in the Figure 4.

Definition 8. Consider $\mathbb{P}_{0} \equiv \mathbb{D}_{0} \amalg \overline{\mathbb{D}}_{0} \amalg \overline{\mathbb{D}}_{0}^{\perp} \equiv\left(\operatorname{Coh}\left(\mathcal{P}_{0}, \approx_{\amalg}\right)\right.$, $\left.\subseteq\right)$ as the amalgamated (smash) sum [7] of $\mathbb{D}_{0}, \overline{\mathbb{D}}_{0}$ and $\overline{\mathbb{D}}_{0}^{\perp}$. The web $\mathbf{P}_{0} \equiv\left(\mathcal{P}_{0}, \approx_{\amalg}\right)$ is defined by the disjoint union $\mathcal{P}_{0} \equiv \mathcal{D}_{0} \cup \dot{\bigcup} \overline{\mathcal{D}}_{0} \cup \overline{\mathcal{D}}_{0}=\left(\{0\} \times \mathcal{D}_{0}\right) \bigcup\left(\{1\} \times \overline{\mathcal{D}}_{0}\right) \bigcup\left(\{2\} \times \overline{\mathcal{D}}_{0}\right)$ together with the coherence relation $\approx_{\amalg}$. In this case, $a_{\alpha} \approx_{\amalg} b_{\beta}$ means that $\alpha=\beta=0$ and $a \approx_{\mathbf{D}_{0}} b$, or $\alpha=\beta=1$ and $a \approx_{\overline{\mathbf{D}}_{0}} b$, or $\alpha=\beta=2$ and $a \approx_{\overline{\mathbf{D}}_{0}^{\perp}} b$.

Definition 9. Let $\mathbf{P}_{0} \prod \mathbf{P}_{0}$ be the web defined by the disjoint union $\left(\mathcal{P}_{0} \cup \mathcal{P}_{0}\right)$ together with the relation $\approx_{\Pi}$ given by $\mathbf{a}_{\alpha} \approx_{\Pi} \mathbf{b}_{\beta} \Leftrightarrow \alpha=\beta, \mathbf{a} \approx_{\amalg} \mathbf{b}$ or $\alpha \neq \beta$, $\forall \mathbf{a}, \mathbf{b} \in \mathcal{D}_{0} \cup \overline{\mathcal{D}}_{0}$. The direct product $\mathbb{P}_{0} \prod \mathbb{P}_{0}$ is the coherence space of sequential product of two (parallel or non-deterministic) elementary processes, whose execution is performed in 2 uct.

Now, we assume a domain of Boolean tests since the deterministic sums of processes are concerned with binary logic for tests. The model, however, admits a more general version with multi-valued logics, which we don't follow here.

Definition 10. Let $\mathbf{B} \equiv\left(\mathcal{B}, \cong_{\mathcal{B}}\right)$ be the discrete web defined by the family $\mathcal{B}$ of all Boolean tests. The coherence space of tests is denoted by $\mathbb{B} \equiv(\operatorname{Coh}(\mathbf{B}), \subseteq)$. The coherence space $\mathbb{P}_{0} \prod_{\mathbb{B}} \mathbb{P}_{0}$ of the deterministic sums of elementary processes, performed in 2 uct, is defined as the direct product between $\mathbb{B}$ and $\mathbb{P}_{0} \prod \mathbb{P}_{0}$. Thus, $\forall \mathbf{a}, \mathbf{b} \in \mathbf{P}_{0}$, the expression $\mathbf{a}_{\alpha} \approx_{\prod_{\mathbb{B}}} \mathbf{b}_{\beta}$ means that
(a) $\alpha=\beta=2$ and $\mathbf{a} \approx_{\mathbb{B}} \mathbf{b}$, or
(b) $\alpha=\beta=1$ and $\mathbf{a} \approx_{\mathbb{P}_{0} \prod \mathbb{P}_{0}} \mathbf{b}$, or
(c) $\alpha=\beta=0$ and $\mathbf{a} \approx_{\mathbb{P}_{0} \prod \mathbb{P}_{0}} \mathbf{b}$, or
(d) $\alpha \neq \beta$.

We can put all the above together, to get:

Definition 11. The domain $\mathbb{D}_{1} \equiv\left(\operatorname{Coh}\left(\mathbf{D}_{1}\right), \subseteq\right)$ is defined by the smash sum

$$
\mathbb{D}_{1}=\mathbb{P}_{0} \amalg\left(\mathbb{P}_{0} \prod \mathbb{P}_{0}\right) \amalg\left(\mathbb{P}_{0} \prod_{\mathbb{B}} \mathbb{P}_{0}\right), \quad \text { and } \quad \mathbb{P}_{0}=\mathbb{D}_{0} \amalg \overline{\mathbb{D}}_{0} \amalg \overline{\mathbb{D}}_{0}^{\perp},
$$

\footnotetext{
${ }^{7} \mathrm{~A}$ unit of computational time is denoted by $u c t$.
} 


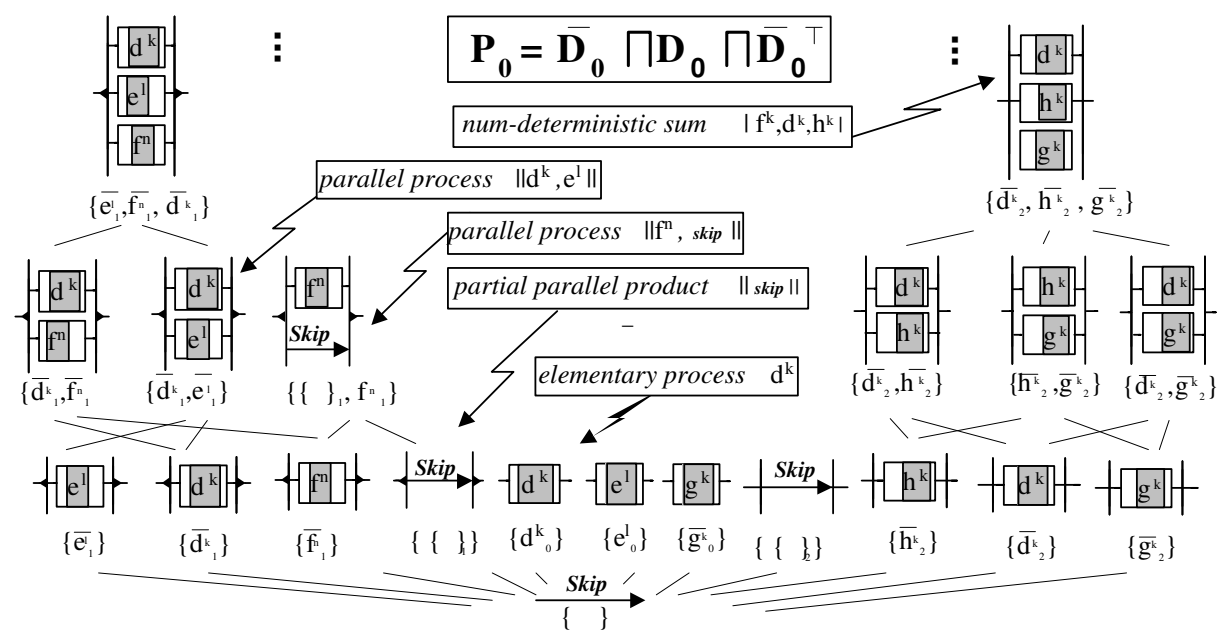

Figure 4: A diagrammatic representation of the coherence space $\mathbb{P}_{0}$.

which encompasses the first step of the construction of the ordered structure of the GM model and provides the representations for all GM computational processes performed in at most 2 uct.

The ideas presented until here can be generalized to the domain

$$
\mathbb{D}_{n+1}=\mathbb{P}_{n} \amalg\left(\mathbb{P}_{n} \prod \mathbb{P}_{n}\right) \amalg\left(\mathbb{P}_{n} \prod_{\mathbb{B}} \mathbb{P}_{n}\right), \quad \mathbb{P}_{n}=\mathbb{D}_{n} \amalg \overline{\mathbb{D}}_{n} \amalg \overline{\mathbb{D}}_{n}^{\perp}
$$

Each indexed token in such domain can present two or tree symbols in its index. The leftmost symbol of an index indicates the one of the following constructors (0) (for the simple inclusion of an element of the previous level in the new one), (1) (indicating the parallel product of elements existing in the previous level) or (2) (indicating the non-deterministic sum of elements existing in the previous level). The second and third symbols, if present, mean the following: that the element is the first (02) or the second (12) summand in a deterministic sum, or that it is the first (01) or the second (11) term in a sequential product. The position information induced by $I$ on the domain of elementary processes can be lifted to the coherent sets of constructed domains by the position-function defined below, which defines the concurrence and conflict relations in such domains.

Definition 12. Consider the index $\alpha \in\{0,1,2\}$, the elementary position-function $\Upsilon_{\mathbb{D}_{0}}: \mathbb{D}_{0} \rightarrow \wp(I), \quad \Upsilon_{\mathbb{D}_{0}}(x)=\left\{i_{k} \mid d^{(k)} \in x\right\}$, together with the following functions:

1. $v_{\mathbf{P}_{0}}: \mathbf{P}_{0} \rightarrow \wp(I), \quad v_{\mathbf{P}_{0}}\left(\mathbf{a}_{0}\right)=\Upsilon_{\mathbb{D}_{0}}(\mathbf{a})$ and $v_{\mathbf{P}_{0}}\left(\mathbf{a}_{\alpha(\alpha \neq 0)}\right)=\left\{i_{k} \mid \overline{d^{(k)}}=\mathbf{a}\right\}$;

2. $\Upsilon_{\mathbb{P}_{0}}: \mathbb{P}_{0} \rightarrow \wp(\omega), \quad \Upsilon_{\mathbb{P}_{0}}(x)=\cup v_{\mathbf{P}_{0}}(\mathbf{a}), \forall \mathbf{a}_{\alpha} \in x ;$

3. $\Upsilon_{\mathbb{P}_{0} \Pi \mathbb{P}_{0}}: \mathbb{P}_{0} \prod \mathbb{P}_{0} \rightarrow \wp(\omega), \quad \Upsilon_{\mathbb{P}_{0} \Pi \mathbb{P}_{0}}(x)=\cup \Upsilon_{\mathbb{P}_{0}}(\mathbf{a}), \forall \mathbf{a}_{\alpha} \in x ;$

4. $\Upsilon_{\mathbb{B}}: \mathbb{P}_{0} \prod_{\mathbb{B}} \mathbb{P}_{0} \rightarrow \wp(\omega), \quad \Upsilon_{\mathbb{B}}(x)=\cup \Upsilon_{\mathrm{P}_{0}}(\mathbf{a}), \forall \mathbf{a}_{\alpha(\alpha \neq 2)} \in x$.

Then, $\Upsilon_{\mathbb{D}_{n+1}}: \mathbb{D}_{n+1} \rightarrow \wp(I)$ is given by cases in the following expressions 
(a) $\Upsilon_{\mathbf{D}_{n+1}}(x)=\cup \Upsilon_{\mathbf{P}_{n}}(\mathbf{a}), \forall \mathbf{a}_{0} \in x$, or $(b) \Upsilon_{\mathbf{D}_{n+1}}(x)=\cup \Upsilon_{\mathbf{P}_{n} \Pi \mathbf{P}_{n}}(\mathbf{a}), \forall \mathbf{a}_{1} \in x$, or (c) $\Upsilon_{\mathbf{D}_{n+1}}(x)=\cup \Upsilon_{\mathbf{P}_{n} \Pi_{\mathbf{B}} \mathbf{P}_{n}}(\mathbf{a}), \forall \mathbf{a}_{2} \in x$.

Definition 13. Consider the indexes $\beta \in\{0,1\}, \theta \in\{0,1,2\}$ to introduce the embedding-functions and related projections (represented in the Figure 3 )

1. $(a) \gamma_{n}^{(0)}, \gamma_{n}^{(1)}, \gamma_{n}^{(2)}: \mathbb{P}_{n}, \mathbb{P}_{n} \prod \mathbb{P}_{n}, \mathbb{P}_{n} \prod_{\mathbb{B}} \mathbb{P}_{n} \rightarrow \mathbb{D}_{n+1}, \gamma_{n}^{(\theta)}(x)=\left\{\mathbf{a}_{\alpha} \mid \mathbf{a} \in x\right\} ;$ $(b) \Gamma_{n}^{(0)}, \Gamma_{n}^{(1)}, \Gamma_{n}^{(2)}: \mathbb{D}_{n+1} \rightarrow \mathbb{P}_{n}, \mathbb{P}_{n} \prod \mathbb{P}_{n}, \mathbb{P}_{n} \prod_{\mathbb{B}} \mathbb{P}_{n}, \Gamma_{n}^{(\theta)}(x)=\left\{\mathbf{a} \mid \mathbf{a}_{\alpha} \in x\right\}$

2. $(a) \phi_{n}^{(0)}, \phi_{n}^{(1)}, \phi_{n}^{(2)}: \mathbb{D}_{n}, \overline{\mathbb{D}}_{n}, \overline{\mathbb{D}}^{\perp} \rightarrow \mathbb{P}_{n}, \phi_{n}^{(\theta)}(x)=\left\{\mathbf{a}_{\theta} \mid \mathbf{a} \in x\right\}$;

(b) $\Phi_{n}^{(0)}, \Phi_{n}^{(1)}, \Phi_{n}^{(2)}: \mathbb{P}_{n} \rightarrow \mathbb{D}_{n}, \overline{\mathbb{D}}_{n}, \overline{\mathbb{D}}^{\perp}, \quad \Phi_{n}^{(\theta)}(x)=\left\{\mathbf{a} \mid \mathbf{a}_{\theta} \in x\right\}$.

3. $\left(\right.$ a) $\psi_{n}^{(\beta)}: \mathbb{D}_{n} \rightarrow \mathbb{P}_{n} \prod \mathbb{P}_{n}, \quad \psi_{n}^{(\beta)}(x)=\left\{\mathbf{a}_{\beta} \mid \mathbf{a} \in x\right\}$;

(b) $\Psi_{n}^{(\beta)}: \mathbb{P}_{n} \prod \mathbb{P}_{n} \rightarrow \mathbb{D}_{n}, \quad \Psi_{n}^{(\beta)}(x)=\left\{\mathbf{a} \mid \mathbf{a}_{\beta} \in x\right\}$.

4. (a) $\lambda_{n}^{(0)}, \lambda_{n}^{(1)}, \lambda_{n}^{(2)}: \mathbb{P}_{n}, \mathbb{P}_{n}, \mathbb{B} \rightarrow \mathbb{P}_{n} \prod_{\mathbb{B}} \mathbb{P}_{n}, \quad \lambda_{n}^{(\theta)}(x)=\left\{\mathbf{a}_{\theta} \mid \mathbf{a} \in x\right\}$.

(b) $\Lambda_{n}^{(0)}, \Lambda_{n}^{(1)}, \Lambda_{n}^{(2)}: \mathbb{P}_{n} \prod_{\mathbb{B}} \mathbb{P}_{n} \rightarrow \mathbb{P}_{n}, \mathbb{P}_{n}, \mathbb{B}, \quad \Lambda_{n}^{(\theta)}(x)=\left\{\mathbf{a} \mid \mathbf{a}_{\theta} \in x\right\}$.

Definition 14. Let $\theta \in\{0,1,2\}$ be an index and consider ${ }^{8}$

- $\pi_{n}^{(0)}, \pi_{n}^{\leftarrow(0)} ; \pi_{n}^{(1)}, \pi_{n}^{\leftarrow(1)} ; \pi_{n}^{(2)}, \pi_{n}^{\leftarrow(2)}: \mathbb{D}_{n} ; \overline{\mathbb{D}}_{n} ; \overline{\mathbb{D}}_{n}^{\perp} \rightarrow \mathbb{D}_{n+1}$, and

- $\Pi_{n}^{\left({ }^{(0)}\right.}, \Pi_{n}^{\leftarrow(0)} ; \Pi_{n}^{(1)}, \Pi_{n}^{\leftarrow(1)} ; \Pi_{n}^{\rightarrow(2)}, \Pi_{n}^{\leftarrow(2)}: \mathbb{D}_{n+1} \rightarrow \mathbb{D}_{n} ; \overline{\mathbb{D}}_{n} ; \overline{\mathbb{D}}_{n}^{\perp}$,

as embedding-functions and related projections, whose definitions are given by

1. if $x \in \gamma_{n}^{(0)}\left[\mathbb{P}_{n}\right]$ then

$$
(a) \pi_{n+1}^{\rightarrow(\theta)}(x)=\gamma_{n}^{(0)} \circ \phi_{n}^{(\theta)}=\pi_{n+1}^{\leftarrow(\theta)}(x), \quad(b) \Pi_{n+1}^{\rightarrow(\theta)}(x)=\Phi_{n}^{(\theta)} \circ \Gamma_{n}^{(0)}=\Pi_{n+1}^{\leftarrow(\theta)}(x) ;
$$

2. if $x \in \gamma_{n}^{(1)}\left[\mathbb{P}_{n} \prod \mathbb{P}_{n}\right]$ then
(a) $\pi_{n+1}^{\rightarrow(\theta)}=\gamma_{n}^{(1)} \circ \psi_{n}^{(0)} \circ \phi_{n}^{(\theta)}, \pi_{n+1}^{\leftarrow(\theta)}=\gamma_{n}^{(1)} \circ \psi_{n}^{(1)} \circ \phi_{n}^{(\theta)}$, and
(b) $\Pi_{n+1}^{\rightarrow(\theta)}=\Phi_{n}^{(\theta)} \circ \Psi_{n}^{(0)} \circ \Gamma_{n}^{(1)}, \Pi_{n+1}^{\leftarrow(\theta)}=\Phi_{n}^{(\theta)} \circ \Psi_{n}^{(1)} \circ \Gamma_{n}^{(1)}$;

3. if $x \in \gamma_{n}^{(2)}\left[\mathbb{P}_{n} \prod_{\mathbb{B}} \mathbb{P}_{n}\right]$ then
(a) $\pi_{n+1}^{\rightarrow(\theta)}(x)=\gamma_{n}^{(2)} \circ \psi_{n}^{(0)} \circ \phi_{n}^{(\theta)}, \pi_{n+1}^{\leftarrow(\theta)}(x)=\gamma_{n}^{(2)} \circ \psi_{n}^{(1)} \circ \phi_{n}^{(\theta)}$ and
(b) $\Pi_{n+1}^{\rightarrow(\theta)}(x)=\Phi_{n}^{(\theta)} \circ \Psi_{n}^{(0)} \circ \Gamma_{n}^{(2)}, \Pi_{n+1}^{\leftarrow(\theta)}(x)=\Phi_{n}^{(\theta)} \circ \Psi_{n}^{(1)} \circ \Gamma_{n}^{(2)}$.

When $n=0, \pi_{0}^{\rightarrow(\theta)}=\pi_{0}^{\leftarrow(\theta)}=\left\{\mathbf{a}_{\theta 0} \mid \mathbf{a} \in x\right\}$ and $\Pi_{0}^{\rightarrow(\theta)}=\Pi_{0}^{\leftarrow(\theta)}=\left\{\mathbf{a} \mid \mathbf{a}_{\theta 0} \in x\right\}$.

In particular, $\pi_{n}^{(\theta)}(\emptyset)=\pi_{n}^{\leftarrow(\theta)}(\emptyset)=\emptyset$ and $\Pi_{n}^{(\theta)}(\emptyset)=\Pi_{n}^{\leftarrow(\theta)}(\emptyset)=\emptyset$.

Proposition 1. The embedding and projection-functions presented in Definitions 13, 14 are linear functions. ${ }^{9}$ In addition, $\left(\mathbb{D}_{n}, \mathbb{D}_{n+1}\right)$ is a projection-pair in the category $\operatorname{Cosp} \mathcal{L}$ in, that is $\pi_{n} \circ \Pi_{n}^{\rightarrow} \subseteq \mathbb{D}_{n+1}$ and $\Pi_{n} \circ \pi_{n}=\mathbb{D}_{n}$ hold.

In the end of this section, the completion of the domain $\mathbb{D}_{\infty}$ is presented.

\footnotetext{
${ }^{8}$ The notations $\pi_{n}^{(0)} \equiv \pi_{n}$ and $\Pi_{n}{ }^{(0)} \equiv \Pi_{n}$ are assumed.

${ }^{9}$ The proves of propositions and theorems of this paper can be found in [4].
} 
Definition 15. The coherence space $\mathbb{D}_{\infty} \rightarrow$ of computational processes of the GM model is defined as the least fixed point for the equation $(*)$. That is,

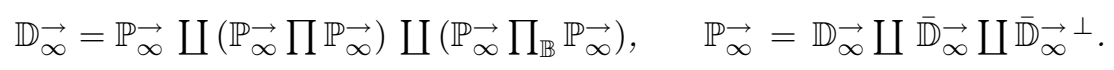

In order to prove that $(*)$ has a least fixed point, consider the notation $\mathbf{a}_{: \theta} \equiv$ $\mathbf{a}_{\theta . \theta} \ldots$ and the following. Let $\mathcal{K} \rightarrow$ be a diagram defined by the family of coherence spaces $\left\{\mathbb{D}_{n}\right\}_{n \in \omega}$ and projections $\Pi_{n}: \mathbb{D}_{n+1} \rightarrow \mathbb{D}_{n}$, represented by

$$
\mathbb{D}_{0} \stackrel{\Pi_{1}^{\rightarrow}}{\longleftarrow} \mathbb{D}_{1} \longleftarrow \ldots \longleftarrow \mathbb{D}_{n} \stackrel{\Pi_{n+1}^{\rightarrow}}{\longleftarrow} \mathbb{D}_{n+1} \longleftarrow \ldots
$$

By Definition 14, for each $w_{n+1} \in \mathbb{D}_{n+1}$, there is $w_{n}=\Pi_{n}\left(w_{n+1}\right) \in \mathbb{D}_{n}$. In the next definition, for each subset $\left\{w_{n}\right\}_{n \in \omega}$ there is another $\left\{x_{n}\right\}_{n \in \omega} \subseteq \mathbb{D}_{\infty}$,

$$
x=\bigcup_{n \in \omega}^{\uparrow}\left\{x_{n}\right\} \quad \Leftrightarrow \quad x_{0} \subseteq x_{1} \subseteq \ldots \subseteq x_{n} \subseteq \ldots \subseteq x \text { and } \Pi_{\infty, n}^{\rightarrow}\left(x_{n}\right)=w_{n} .
$$

Definition 16. $\pi_{n, \infty}^{\rightarrow}: \mathbb{D}_{n} \rightarrow \mathbb{D}_{\infty}$ is given by $\pi_{0, \infty}(x)=\left\{\mathbf{a}_{: 00} \mid \mathbf{a} \in x\right\}$, and

(a) $\pi_{n+1, \infty}^{\rightarrow}(x)=\left\{\mathbf{a}_{: 00} \mid \mathbf{a} \in x\right\}$, if $x \in \gamma_{n}^{(0)}\left[\mathbb{P}_{n}\right]$,

(b) $\pi_{n+1, \infty}^{\vec{m}}(x)=\left\{\mathbf{a}_{: 001} \mid \mathbf{a} \in x\right\}$, if $x \in \gamma_{n}^{(1)}\left[\mathbb{P}_{n} \prod \mathbb{P}_{n}\right]$,

(c) $\pi_{n+1, \infty}^{\rightarrow}(x)=\left\{\mathbf{a}_{: 002} \mid \mathbf{a} \in x\right\}$, if $x \in \gamma_{n}^{(2)}\left[\mathbb{P}_{n} \prod_{\mathbb{B}} \mathbb{P}_{n}\right]$.

Now, let $x=\bigcup_{m \in \omega}^{\uparrow}\left\{x_{m} \mid x_{m} \in \pi_{m, \infty}^{\rightarrow}\left[\mathbb{D}_{m}\right]\right\}$ be a directed subset of finite approximations of $x$. The projection-function $\Pi_{\infty, n}: \mathbb{D}_{\infty} \rightarrow \mathbb{D}_{n}$ is given by $\Pi_{\infty, n+1}(x)=$ $\bigcup_{m \in \omega}^{\uparrow}\left\{\Pi_{m, n} \circ \Pi_{\infty, m}^{\rightarrow}\left(x_{m}\right)\right\}$ where $\Pi_{\infty, 0}^{\rightarrow}\left(x_{m}\right)=\left\{\mathbf{d}^{k} \mid \mathbf{d}_{: 00}^{k}=x_{m}\right\}$ and

(a) $\Pi_{\infty, m}^{\rightarrow}\left(x_{m}\right)=\left\{\mathbf{a} \mid \mathbf{a}_{: 00} \in x_{m}\right\}$, if $x_{m} \in\left(\pi_{m, \infty} \circ \gamma_{m-1}^{(0)}\right)\left[\mathbb{P}_{m-1}\right]$,

(b) $\Pi_{\infty, m}^{\vec{\infty}}\left(x_{m}\right)=\left\{\mathbf{a} \mid \mathbf{a}_{: 001} \in x_{m}\right\}$, if $x_{m} \in\left(\pi_{m, \infty}^{\vec{\infty}} \circ \gamma_{m-1}^{(1)}\right)\left[\mathbb{P}_{m-1} \prod \mathbb{P}_{m-1}\right]$,

(c) $\Pi_{\infty, m}^{\rightarrow}\left(x_{m}\right)=\left\{\mathbf{a} \mid \mathbf{a}_{: 002} \in x_{m}\right\}$, if $x_{m} \in\left(\pi_{m, \infty}^{\rightarrow} \circ \gamma_{m-1}^{(2)}\right)\left[\mathbb{P}_{m-1} \prod_{\mathbb{B}} \mathbb{P}_{m-1}\right]$.

Proposition 2. The projection and embedding-functions $\Pi_{\infty, n}^{\rightarrow}$ and $\pi_{\infty, n}$ are linear functions. Moreover, $\Pi_{\infty, n}^{\overrightarrow{ } \circ \pi_{\infty, n}}=\mathbb{D}_{n}$ and $\pi_{\infty, n} \circ \Pi_{\infty, n} \subseteq \mathbb{D}_{\infty}^{\rightarrow}$, which guarantees $\left(\mathbb{D}_{\infty}, \mathbb{D}_{n}\right)$ as a projection-pair in the $\mathcal{C} \operatorname{osp} \mathcal{L}$ in category.

Theorem 1. The pair $\left(\mathbb{D}_{\infty}, \Pi_{\infty, n}: \mathbb{D}_{\infty} \rightarrow \mathbb{D}_{n}\right)$ is a limit cone for diagram $\mathcal{K} \rightarrow$.

According Theorem 1 , the domain $\mathbb{D}_{\infty}$ is the least fixed point of the equation (*) that defines the representation of finite processes. The inductive nature of the model and its completion procedure ensure the existence of the interpretation for the recursive constructors. Therefore, this model is able to interpret processes that have no restrictions related to memory size, and it can be completed for temporally infinite sequential processes. The latter can be done in many different ways, generating many different time-lines, such as the usual $0,1, \ldots+\infty,-\infty \ldots,-1,0$, $-\infty \ldots,-1,0,+1, \ldots+\infty$, but also ones such as $0,1, \ldots-2,-1$. That corresponds to the use of transfinite ordinals to represent time.

In this sense, the Figure 5 (a) shows the representation of partial objets in the related coherence spaces $\mathbb{D}_{\infty}^{\rightarrow}, \mathbb{D}_{\infty}^{\leftarrow}, \mathbb{D}_{\infty}^{\rightarrow \leftarrow}$ and $\mathbb{D}_{\infty}^{\leftrightarrow}$. The domains $\mathbb{D}_{\infty}^{\rightarrow}$ and $\mathbb{D}_{\infty}^{\leftarrow}$ contain the prefixed and suffixed sequential constructions, respectively. In the same way, the coherence space $\mathbb{D}_{\infty}^{\leftrightarrow}$ contains infixed sequential constructions. 


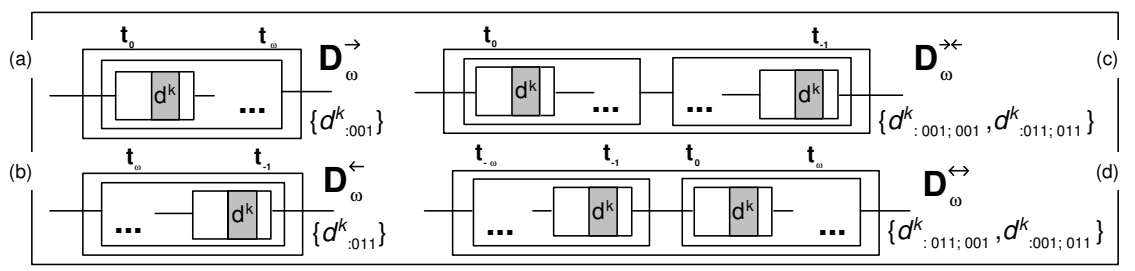

Figure 5: Exemplifications of objects in different ordered structures of GM models.

In the following, we note that the concurrence relation defined over the processes in $\mathbb{D}_{\infty}$ is induced by the position-function $\Upsilon_{\mathbb{D}_{\infty}}$, presented in the next definition.

Definition 17. Consider $x$ such that $x=\bigcup_{i \in \omega}^{\uparrow}\left\{x_{i}\right\} \in \mathbb{D}_{\infty}^{\rightarrow}, x_{i} \in \pi_{n, \infty}\left[\mathbb{D}_{n}\right]$ and the function $\Upsilon_{\mathbb{D}_{n}}$ in the Definition 12. The position-function $\Upsilon_{\mathbb{D}_{\infty}}: \mathbb{D}_{\infty}^{\rightarrow} \rightarrow \wp(\omega)$ is defined as $\Upsilon_{\mathbb{D}_{\infty}}(x)=\bigcup \Upsilon_{\mathbb{D}_{n}}\left[\Pi_{\infty, n}(x)\right]=\bigcup \Upsilon_{\mathbb{D}_{n}}\left[\bigcup_{i \in \omega}^{\uparrow} \Pi_{\infty, n}\left(x_{i}\right)\right]$.

Definition 18. Let I be the set of labels of a geometric space, $V$ be the set of values and $\mathcal{A}$ be the set of action. The GM model is defined by the system

$$
G M \equiv\left(\mathbb{S}, \mathbb{D}_{\infty}, \mathbb{B}\right),
$$

where $\mathbb{S}$ is the coherence space of computational states, $\mathbb{D}_{\infty}$ is the coherence space of computational processes and $\mathbb{B}$ is the coherence space of computational text, previously presented in the above sections.

\section{Conclusions}

The inductive nature of the model and its completion ensure the existence of the interpretation for recursive constructors, both in the conceptual and in the temporal senses of such construction. Therefore, this model is able to interpret processes without restrictions related to memory space, and it can be completed for temporary infinite processes.

In addition, the study of the behavior of the modelled processes can be characterized the semantics of this model, based on the analysis of objects and morphism in the category $\operatorname{Cosp} \mathcal{L}$ in. The related language, inherent to the representations in $\mathbb{D}_{\infty}$, gives the operational representation of the usual algebraic process constructors.

A more generic version of GM model can be also constructed, with a transfinite global memory shared by synchronized processes distributed over an enumerable set of geometric machines. That model is formalized by the coherence space of transfinite computational processes, defined over a web of tokens indexed by transfinite ordinal numbers.

Finally, this model can be applied, to various kinds of computations involving array structures, such as matrix computations and cellular automata. Following this approach, it is possible to introduce the interval version the de GM model, 
where the set of values in the GM memory is represented by the coherence space of rational intervals $\mathbb{I} \mathbb{Q}[1]$, a constructive computational representation of the the space of real intervals. In particular, a semantic modelling of interval algorithms related to the arithmetic operations can be obtained as an application algebraic process constructors.

Resumo. Este trabalho introduz a Máquina Geométrica (MG) - um modelo computacional para construção e representação de processos concorrentes e nãodeterminísticos, executados de forma sincronizada, com memória infinita cujas posições são rotuladas por pontos de um espaço geométrico. A estrutura ordenada do modelo MG é formalizada como um Espaço Coerente de Girard. A partir do espaço coerente de procesos elementares, a estrutura indutiva deste domínio é sistematicamente construída em etapas e o procedimento de completação assegura a existência de computações temporal e espacialmente infinitas. Um especial objetivo deste trabalho é aplicar esta representação baseada em espaços coerentes na modelagem semântica de computações paralelas e distribuídas, definidas sobre estruturas matriciais.

\section{References}

[1] G.P. Dimuro, A.C.R. Costa and D.M. Claudio, A coherence space of rational intervals for a construction of IR, Journal of Reliable Computing, 6 (2000), 139-178.

[2] J.-Y. Girard, The system F of variable types, fifteen years later, Theoretical Computer Science, 45 (1986), 159-192.

[3] J.-Y. Girard, Linear logic, Theoretical Computer Science, 1 (1987), 187-212.

[4] R.H.S. Reiser, "The Geometric Machine - a Computational Model for Concurrence and Non-determinism Based on Coherence Spaces", Ph.D. Thesis (in portuguese), PPGC, UFRGS, Porto Alegre, RS, Brazil, 2002. (avaliable in http://gmc.ucpel.tche.br/imqd)

[5] D. Scott, Some definitional suggestions for automata theory, Journal of Computer and System Sciences, 188 (1967), 311-372.

[6] D. Scott, The lattice of flow diagrams, Lecture Notes in Mathematics, 188 (1971), 311-372.

[7] A.S. Troeltra, Lectures on Linear Logic, in "CSLI Lecture Notes", Vol. 29, 1992. 